\title{
Variational Principles and Spatially-Homogeneous Universes, Including Rotation*
}

\author{
M. A. H. MaCCAllum ${ }^{\star}$ and A. H. TAub \\ Department of Mathematics, University of California, Berkeley, Calif., USA
}

Received November 20, 1971

\begin{abstract}
The validity of imposing spatial homogeneity on the variations in the usual action principle for Einstein's equations is studied. It is proved that with this procedure the standard and ADM Lagrangians give correct Einstein equations if and only if the space belongs to Class A of Ellis and MacCallum [1], i.e., the structure constants of the simply transitive group satisfy $\mathrm{C}_{f g}^{f}=0$. The possibility of overcoming this difficulty in the Class $\mathrm{B}$ spaces is examined.
\end{abstract}

\section{Introduction}

When the source of the gravitational field described by the metric tensor ${ }^{4} g_{\mu \nu}$ of space-time is a perfect fluid with stress-energy tensor ${ }^{1}$

$$
T^{\mu v}=(w+p) u^{\mu} u^{v}+p^{4} g^{\mu v}
$$

where $p$ is the pressure, $w$ the energy density and $u^{\mu}$ the fluid velocity $\left(u^{\mu} u_{\mu}=-1\right)$ and when there is an equation of state of the form

$$
w=w(p)
$$

* This work was supported in part by the United States Atomic Energy Commission under Contract Number AT 104-37-39 Project Agreement No. 125.

$\star \star$ Permanent address: Department of Applied Mathematics and Theoretical Physics, University of Cambridge, Silver Street, Cambridge, U. K. and King's College, Cambridge, U. K.

${ }^{1}$ We employ the following conventions. The signature of spacetime is +2 and units are such that $8 \pi G=c=1$. The metric of space-time is denoted ${ }^{4} g_{\mu v}$, the curvature and Einstein tensors by the usual $R_{\mu v}, G_{\mu \nu}$ etc., and covariant differentiation by a semi-colon separating indices. The corresponding quantities for an embedded three-space will be denoted by $g_{i j} ; R^{*}{ }_{i j}, G^{*}{ }_{i j}$ etc.; and a bar separating indices. Greek indices run from 1 to 4 , Latin indices from 1 to $3, x^{4}$ being a time coordinate. A comma separating indices denotes partial derivative. Any undefined notation may be assumed to have its standard meaning. The sign conventions for the Riemann and Ricci tensors follow [1], i.e., for an arbitrary vector $b^{v}$

$$
\begin{gathered}
b_{; \mu \tau}^{v}-b_{; \tau \mu}^{v}=-R_{\sigma \mu \tau}^{v} b^{\sigma}, \\
R_{\sigma \tau}=R_{\sigma \nu \tau}^{v} ; R=R_{\sigma}^{\sigma} .
\end{gathered}
$$


we may use co-moving coordinates to reduce the number of dependent variables occurring in the Einstein field equations. We define the function $s(w)($ or $s(p))$ by the equation

$$
\frac{d s}{s}=\frac{d w}{w+p} .
$$

Comoving coordinates may be chosen so that [2]

where

$$
u^{\mu}=e^{-\phi} \delta_{4}^{\mu}
$$

and

$$
\begin{aligned}
s e^{-\phi} & =w+p=f\left(x^{i}\right) / \sqrt{-{ }^{4} g} . \\
u_{\mu} & =e^{-\phi 4} g_{\mu 4}=e^{\phi} V_{\mu}\left(x^{i}\right)
\end{aligned}
$$

where

$$
V_{4}=-1, V_{i}=V_{i}\left(x^{j}\right), \quad i, j=1,2,3 .
$$

The Einstein field equations ${ }^{2}$

$$
R_{\mu \nu}-\frac{1}{2} R^{4} g_{\mu \nu}=T_{\mu \nu}
$$

then become partial differential equations for the functions $V_{i}$ which depend only on the $x^{j}$, the space coordinates, for the functions

$$
{ }^{4} g_{i j}=g_{i j}\left(x^{4}, x^{k}\right), \quad k=1,2,3
$$

and for the function

$$
\phi=\phi\left(x^{4}, x^{k}\right) .
$$

These equations may be derived from the usual variational principle based on the action integral

$$
{ }^{4} I=\int_{V}(R+2 p(\phi)) \sqrt{-{ }^{4} g} d^{4} x
$$

where $R$ is the scalar curvature of space-time and ${ }^{4} g=\operatorname{det}^{4} g_{\mu v}$.

When the space-time is spatially homogeneous, that is, admits a three-parameter simply-transitive group of motions, these Einstein field equations may be reduced to ordinary differential equations for functions $\phi\left(x^{4}\right)$ and $\gamma_{a b}\left(x^{4}\right)$, simply related to the functions listed above. In this case the action integral reads

$$
{ }^{4} I=\int \mathscr{L}\left(x^{4}\right) d x^{4} \int L\left(x^{i}\right) d^{3} x .
$$

It is the purpose of this paper to determine the conditions under which the Einstein equations for $\phi$ and $\gamma_{a b}$ are the Euler equations derived from the action integral

$$
I=\int \mathscr{L}\left(x^{4}\right) d x^{4} .
$$

2 The cosmological constant, which does not affect the issues under discussion here, is omitted for brevity's sake only. 
In other words we wish to discover when the operation of deriving the Euler equations commutes with the imposition of a symmetry condition, namely spatial homogeneity.

It is not necessarily valid to impose the symmetry before taking the variations. To see this, consider varying a Lagrangian of the form

$$
I=\int L\left(\phi^{A} ; \phi^{A}{ }_{, \mu}\right) d^{4} x
$$

where $\phi^{A}(A=1, \ldots, n)$ are some fields and $\phi^{A}{ }_{, \mu}$ their derivatives. One obtains

$$
I^{\prime}=\int\left(\frac{\partial L}{\partial \phi^{A}}-\frac{\partial}{\partial x^{\mu}}\left(\frac{\partial L}{\partial \phi^{A}{ }_{, \mu}}\right)\right) \phi^{\prime A} d^{4} x+\int\left(\frac{\partial L}{\partial \phi^{A}{ }_{, \mu}} \phi^{\prime A}\right)_{, \mu} d^{4} x
$$

where the variation has been taken by introducing a one-parameter family $\phi^{A}(x, e)$ and differentiating with respect to the parameter $e$ (' denotes $d / d e$ ). The last term in (1.11) is made zero by imposing certain boundary conditions on the $\phi^{A \prime}$. (The alternative of taking the "natural" boundary conditions on $\frac{\partial L}{\partial \phi^{A}, \mu}$ is not very useful for local field theories.) Thus one deduces the Euler-Lagrange equations

$$
\frac{\partial L}{\partial \phi^{A}}-\frac{\partial}{\partial x^{\mu}}\left(\frac{\partial L}{\partial \phi_{, \mu}^{A}}\right)=0
$$

from $I^{\prime}=0$ by considering the set of all variations satisfying specified boundary conditions. However, when the variations are required to satisfy some symmetry condition, the boundary conditions may, by the symmetry, restrict the variations $\phi^{A \prime}$ in the interior of the region of integration. Alternatively one can say that for an arbitrary variation obeying the symmetry restriction the last expression in (1.11) might not vanish, so that $I^{\prime}=0$ no longer leads to (1.12).

In Section 2 of this paper we give the Einstein equations and variational principle for the general case of fluid obeying (1.1)-(1.2). In Section 3 we give the Einstein equations for the spatially homogeneous case and in Section 4 discuss the validity of a Lagrangian such as (1.9). Section 5 discusses the form of the Lagrangian in those cases where it is valid, and contains some remarks on the effects of rotation. Finally there is a short summary.

\section{Fluid-filled Space-times}

We adopt the stress-energy (1.1) and (1.2). Although (1.2) rules out dust, we assume it only to be able to use a particular matter Lagrangian. Clearly one must obtain the correct dust equations as a limiting case. 
In fact these can be obtained from the specialization to $p=0$ of a Lagrangian which differs from ours but is equivalent to it when (1.2) holds. As a general rule any two Lagrangians yielding the same Einstein equations are equivalent, see e.g. [3].

We now employ the coordinates specified by $(1.3)-(1.6)^{3}$. The usual kinematic quantities, as defined by Ehlers [5], i.e., acceleration vector, rotation tensor, vorticity vector, expansion tensor, expansion and shear tensor, are respectively

$$
\left.\begin{array}{rl}
\dot{u}_{\mu} & =\phi_{, \mu}+\phi_{, 4} V_{\mu} \\
\omega_{\mu v} & =e^{\phi} V_{[\mu, v]} \\
\omega^{\mu} & =\frac{1}{2} \eta^{\mu \nu \sigma \tau} u_{v} \omega_{\sigma \tau} ; \omega^{4}=V_{i} \omega^{i} ; \omega^{i}=\frac{-e^{2 \phi}}{2 \sqrt{-{ }^{4} g}} \varepsilon^{i j k} V_{j \mid k} \\
\theta_{\mu \nu} & =\frac{e^{-\phi}}{2}\left(h_{\mu v, 4}\right) \\
\theta & =\frac{\left(e^{-\phi} \sqrt{-{ }^{4} g}\right)_{.4}}{\sqrt{-{ }^{4} g}} \\
\sigma_{\mu \nu} & =\frac{e^{-\phi}}{2}\left(e^{\phi} \sqrt{-{ }^{4} g}\right)^{2 / 3}\left(\frac{h_{\mu v}}{\left(e^{-\phi} \sqrt{-{ }^{4} g}\right)^{2 / 3}}\right)_{, 4}
\end{array}\right\}
$$

where $\cdot$ denotes (covariant) differentiation in the $u^{\mu}$ direction, $h_{\mu \nu}=g_{\mu \nu}$ $+u_{u} u_{v}$ is the operator projecting into the subspace orthogonal to $u^{\mu}$, $\eta^{\mu \nu \sigma \tau}$ is the skew tensor whose components in our frame are given by $\eta^{1234}=\left(-{ }^{4} g\right)^{-1 / 2}$, and $\varepsilon^{i j k}$ is the usual Levi-Civita symbol.

The future-pointing unit normal to the surfaces $t=$ constant is

$$
n_{\mu}=-N \delta_{\mu}^{4} .
$$

In these surfaces $g_{i j}={ }^{4} g_{i j}$. If $g^{i j}$ is the inverse of $g_{i j}$, the operator projecting into the subspace orthogonal to $n^{\mu}$ is

$$
k^{\mu v}={ }^{4} g^{\mu v}+n^{\mu} n^{v}=g^{i j} \delta_{i}^{\mu} \delta_{j}^{v} .
$$

Some other useful relations are

$$
\begin{aligned}
& n^{4}=-N^{4} g^{44}=\frac{1}{N}, \quad n^{k}=-\frac{e^{2 \phi} g^{k j} V_{j}}{N}=: \frac{-p^{k}}{N} \\
& N^{2}=e^{2 \phi}+g^{i j} V_{i} V_{j} e^{4 \phi} ; \quad \sqrt{-4} g=N \sqrt{g} .
\end{aligned}
$$

Here and in the sequel three-space quantities have indices raised and lowered by $g_{i j}, g^{i j}$. The second fundamental form (extrinsic curvature)

${ }^{3}$ The possible further specializations of the $V_{1}[4]$ are not useful in the present context. 
of the three-spaces is

$$
K_{i j}=n_{\mu ; \nu} \delta_{i}^{\mu} \delta_{j}^{v}=\frac{1}{2 N}\left(g_{i j, 4}-p_{i \mid j}-p_{j \mid i}\right) .
$$

The acceleration of $n^{\mu}$ is

$$
n_{\mu ; v} n^{v}=\frac{N_{, \varrho} k_{\mu}^{\varrho}}{N} .
$$

The $(3+1)$ splitting into space and time we have now introduced is essentially the same as that of Arnowitt, Deser and Misner (ADM) [6].

The Gauss and Codazzi-Mainardi equations of the embedded threespaces [7] help us to express the Einstein equations in terms of the threespace quantities. We have

$$
R=R^{*}+\Omega+2 \lambda_{; \sigma}^{\sigma}
$$

where $\Omega_{i j}=K_{i}^{k} K_{k j}-K K_{i j} ; K=K_{i}^{i} ; \Omega=\Omega_{i}^{i} ; \lambda^{\sigma}=\left(K n^{\sigma}-n_{; v}^{\sigma} n^{v}\right)$. The Einstein equations read as follows. The $G^{44}$ equation gives

$$
\frac{1}{2}\left(-R^{*}+\Omega\right)=p-(w+p) N^{2} e^{-2 \phi} ;
$$

the $G^{4}{ }_{j}$ equations are

$$
\left(K_{j}^{k}-K \delta^{k}\right)_{\mid k}=-N(w+p) V_{j}=-\frac{f\left(x^{i}\right) V_{j}}{\sqrt{g}}
$$

(using (1.5) and (2.3)), and the $G_{i j}$ equations are

$$
\begin{aligned}
G^{*}{ }_{i j}-\frac{1}{N}\left(N_{\mid i j}-N^{\mid k}{ }_{k} g_{i j}\right)-2 \Omega_{i j} & -\frac{1}{2} \Omega g_{i j}+\frac{1}{N}\left(K_{i j}-K g_{i j}\right)_{4}+K\left(K_{i j}-K g_{i j}\right) \\
& -\frac{1}{N} \mathscr{L}\left(K_{i j}-K g_{i j}\right)=(w+p) e^{2 \phi} V_{i} V_{j}+p g_{i j}
\end{aligned}
$$

where $\mathscr{L}$ denotes the (three-space) Lie derivative.

One can also write the equation $R^{44}=T^{44}-\frac{1}{2} T^{4} g^{44}$, which is the trace of (2.7) combined with (2.5), as

$$
K_{, \varrho} n^{\varrho}+K_{i}^{j} K_{j}^{i}-\frac{N_{\mid k}{ }^{k}}{N}=\frac{w-p}{2}-(w+p) N^{2} e^{-2 \phi}
$$

which is Raychaudhuri's equation for the normal congruence. Subtracting (2.8) and (2.5) from (2.7) and raising an index, gives

$$
\begin{aligned}
R_{j}^{* i}+\frac{\left(\sqrt{g} K_{j}^{i}\right)_{, 4}}{\sqrt{g} N} & -\frac{1}{N} \underset{p^{k}}{\mathscr{L}} K^{i}{ }_{j}-\frac{N^{\mid i}{ }_{j}}{N}-\frac{p_{l l}^{l} K_{j}^{i}}{N} \\
& =(w+p) e^{2 \phi} V^{i} V_{j}+\left(\frac{w-p}{2}\right) \delta_{j}^{i} .
\end{aligned}
$$


Defining $M_{\mu}{ }^{v}=G_{\mu}{ }^{\nu}-T_{\mu}{ }^{\nu}$, and given $M_{i j}=0$ and $M_{\mu}{ }^{v} ; v=0$, the remaining equations in $M_{\mu}{ }^{v}=0$ appear as first integrals, in that if satisfied initially they are satisfied at all times. For as is well-known $M_{\mu}{ }^{v} ; v=0$ leads to

and to

$$
\begin{aligned}
0= & n_{\mu}\left(M^{\mu \varrho}\right)_{; \varrho}=\left(n_{\mu} M^{\mu \varrho}\right)_{; \varrho}-n_{\mu ; \varrho} M^{\mu \varrho} \\
= & -\left(\left(M^{\mu v} n_{\mu} n_{v}\right) n^{\varrho}\right)_{; \varrho}+\left(n_{\mu} M^{\mu}{ }_{i} g^{i j}\right)_{; j} \\
& +n_{i ; \mu} n^{\mu}\left(n_{v} M_{j}^{v} g^{i j}\right)-K_{i j} M_{k l} g^{i k} g^{j l}
\end{aligned}
$$

$$
\begin{aligned}
\left(-\left(n_{v} M^{v}{ }_{i}\right) n^{\tau} \sqrt{-{ }^{4} g}\right)_{, \tau} & +\left(\sqrt{-{ }^{4} g} M_{i l} g^{l n}\right)_{, n}+\sqrt{-{ }^{4} g}\left(M^{\mu v} n_{\mu} n_{v}\right) n_{\varrho} n^{\varrho}{ }_{, i} \\
& +\sqrt{-{ }^{4} g}\left(n_{v} M^{v}{ }_{k}\right) g^{k l} n^{\varrho}{ }^{4} g_{\varrho k, i} \\
& -\frac{\sqrt{-{ }^{4} g}}{2} M_{k l} g^{k j} g^{l m} g_{j m, i}=0 .
\end{aligned}
$$

The field equations arise from varying the action (1.7), i.e.,

$$
I_{1}=\int_{V}\left(R^{*}+\Omega+2 \lambda_{; \sigma}^{\sigma}+2 p\right) \sqrt{-{ }^{4} g} d^{4} x
$$

in the compact region $V$ bounded by a closed surface $S$. The $R$ term in (1.7) gives rise to the left sides of (2.5)-(2.7) and the $p$ term gives the right sides. Variations are made by considering one-parameter families of space-times differing only in $V$, and $\operatorname{not}^{4}$ on $S$. The variation of $p$ is [2]

$$
p^{\prime}=-\frac{(w+p)^{4} g_{44}^{\prime}}{2^{4} g_{44}}=-(w+p) \phi^{\prime} .
$$

The calculation of the field equations proceeds by varying $N, p^{j}$ and $g_{i j}$ in (2.12), and setting $I^{\prime}=0$ for arbitrary variations ${ }^{5}$ vanishing on $S$.

Since our general procedure involves the vanishing of the variation at the boundaries, any Lagrangian differing from (2.12) by a divergence will give rise to the same Euler-Lagrange equations, even though the new Lagrangian may not be invariantly defined. Two important amended Lagrangians are

$$
I_{2}=\int_{V}\left(R^{*}+\Omega+2 p\right) \sqrt{-{ }^{4} g} d^{4} x
$$

${ }^{4}$ One could vary over the whole space-time if appropriate asymptotic boundary conditions were imposed on the varied quantities and if the action (2.12) remained finite (cf. [6]). However, in spatially homogeneous universes either the space sections are compact or (2.12) is infinite.

${ }^{5}$ As an aid to any reader who wishes to reconstruct this tedious calculation, we note that if $\left.\left\{{ }_{j k}\right\}^{2}\right\}^{*}$ is a (three dimensional) Christoffel symbol of the second kind, then

$$
\begin{aligned}
\left\{j_{k j}\right\}^{* \prime} & =\frac{1}{2} g^{i m}\left[g^{\prime}{ }_{J \mid k}+g_{k m \mid J}^{\prime}-g_{k J \mid m}^{\prime}\right] \\
g^{i j}\left(R_{t, j}{ }^{*}\right)^{\prime} & =\left(g^{i k} g^{\prime l}-g^{i j} g^{k l}\right) g_{i, \mid k l}^{\prime}
\end{aligned}
$$

and similarly for four-space quantities. 


$$
\text { and } \begin{aligned}
I_{3}=\int_{V}\left(\left(R^{*}+\Omega+2 p\right) \sqrt{-{ }^{4} g}\right. & +\left(\sqrt{-{ }^{4} g} \lambda^{4}\right)_{, 4} \\
& \left.-2\left(\left(K^{k j}-K g^{k j}\right) \sqrt{-{ }^{4} g} P_{j}\right)_{, k}\right) d^{4} x
\end{aligned}
$$

the latter being the ADM form [6]. The actions themselves agree with each other when the divergences by which they differ have vanishing integral over $V$.

When we come to consider imposition of symmetry conditions we need to know the form of the divergences appearing in the course of the general derivation, in order to know whether the boundary terms still vanish. Using $I_{2}$ above, the $R^{*}$ term gives rise to the spatial derivative terms

$$
\begin{aligned}
& \int\left(N \sqrt{g}\left(g^{i l} g^{j k}-g^{i j} g^{k l}\right) g_{i j \mid l}^{\prime}\right)_{, k} d^{4} x, \\
& \int\left(N_{, l}\left(g^{i k} g^{j l}-g^{i j} g^{k l}\right) g_{i j}^{\prime} \sqrt{g}\right)_{, k} d^{4} x
\end{aligned}
$$

while the $\Omega$ term gives rise to the spatial divergences

and

$$
\begin{aligned}
& \int-2\left(\sqrt{g}\left(K^{k}{ }_{j}-K \delta^{k}{ }_{j}\right) p^{\prime j}\right)_{. k} d^{4} x, \\
& \int-\left(\sqrt{g}\left(K^{i j}-K g^{i j}\right) p^{k} g^{\prime}{ }_{i j}\right)_{, k} d^{4} x .
\end{aligned}
$$

The additional divergences arising if (2.12) or (2.14) are used are easy to compute as the actions differ only by a divergence, and taking the divergence of a quantity and taking its variation are commuting operations.

\section{Spatially-Homogeneous Universes}

We restrict ourselves to spatially-homogeneous universes admitting a three-parameter simply-transitive group of motions acting on spacelike surfaces of homogeneity (thus omitting only the Case I of Kantowski and Sachs [8]). Let a basis of Killing vectors of the simply-transitive group be denoted by $\xi_{A}(A=1,2,3)^{6}$. Then

$$
\left[\xi_{A}, \xi_{B}\right]=C_{A B}^{C} \xi_{C}
$$

where $[$,$] is the usual commutation operation [7] and the structure$ constants $C_{A B}^{C}$ satisfy the usual Jacobi identities

$$
C_{B\{C}^{A} C^{B}{ }_{D E]}=0 \text {. }
$$

${ }^{6} A, B, C \ldots ; a, b, c \ldots ; i, j, k \ldots$ will be used for components in (respectively) the Killing vector, reciprocal vector and coordinate bases. 
Since the scalars $T^{\mu v} u_{\mu} u_{v}=w$ and $T^{\mu}{ }_{\mu}=3 p-w$, and the vector $u^{\mu}$, must be group invariants, we have

$$
\begin{aligned}
& \boldsymbol{\xi}_{A} p=\boldsymbol{\xi}_{A} w=0 \\
& {\left[\boldsymbol{\xi}_{A}, \boldsymbol{u}\right]=0}
\end{aligned} \text { for all } A .
$$

The comoving coordinates in which (1.4)-(1.6) hold are specified [2] in such a way that $t$ is an invariantly-defined scalar (up to transformations $t \rightarrow A t+B ; A, B$ constants). Thus the ( $t=$ constant) surfaces are surfaces of homogeneity, $\xi_{A}^{4}=0$, and the cosmic time $\tau$ defined by $n_{\mu}=-\tau_{, \mu}$ is a function of $t$ alone. (3.3) leads to

$$
\xi_{A, 4}^{v}=0
$$

and so we need only give the $\xi_{A}^{j}$ in an initial surface. This has been done taking canonical forms of the structure constants [9].

Reciprocal group generators $\boldsymbol{B}_{a}$ tangent to the group orbits which satisfy

$$
\left[\boldsymbol{B}_{a}, \boldsymbol{\xi}_{A}\right]=0, \text { all } a, \text { all } A
$$

in each surface of transitivity, can be chosen to satisfy the initial value condition $\xi_{A}=-\boldsymbol{B}_{a} \delta_{A}^{a}$ at one point. In the initial surface the vectors then obey

$$
\left[\boldsymbol{B}_{a}, \boldsymbol{B}_{b}\right]=C_{a b}^{c} \boldsymbol{B}_{c}
$$

where the $C_{a b}^{c}$ are the same as the $C_{A B}^{C}$. If we propagate the $\boldsymbol{B}_{a}$ by the choice $^{7}$

$$
\left[\boldsymbol{B}_{a}, \boldsymbol{u}\right]=0
$$

all the conditions above, including (3.5), are conserved, and the $B_{a}{ }^{i}$ are independent of $t$. Explicit forms for the $B_{a}{ }^{i}$ can be given [9]. Using the matrix $B^{a}{ }_{k}$ inverse to $B_{a}{ }^{i}$ we can define Ricci rotation coefficients (cf. [1])

satisfying

$$
\begin{aligned}
& \Lambda_{b a}^{c}=B^{c}{ }_{j} B_{a}{ }^{j}{ }_{i i} B_{b}{ }^{i} \\
& \Lambda_{a b}^{c}-\Lambda_{b a}^{c}=C^{c}{ }_{a b} \\
& \Lambda_{a b}^{c}=\frac{1}{2} \gamma^{c}\left(C_{g a b}+C_{b g a}-C_{a b g}\right)
\end{aligned}
$$

where $\gamma_{a b}=B_{a}{ }^{i} B_{b i}$ is the metric in the invariant vector basis, and indices in this basis are raised and lowered by $\gamma_{a b}$ and its inverse matrix $\gamma^{a b}$. We may write (cf. $[1,10]$ )

$$
C^{a}{ }_{b c}=\eta_{b c t} n^{a t}+\delta^{a}{ }_{c} a_{b}-\delta^{a}{ }_{b} a_{c}=\varepsilon_{b c t} m^{a t}+\delta_{c}^{a} a_{b}-\delta_{b}^{a} a_{c}
$$

where $\eta_{b c t}=\sqrt{\gamma} \varepsilon_{b c t}, \gamma=\operatorname{det} \gamma_{a b}, n^{a t}=n^{(a t)}$ and $m^{a t}=m^{(a t)}$. Then

$$
\begin{aligned}
& B^{c}{ }_{i \mid j}=-\Lambda_{a b}^{c} B_{i}^{a} B_{j}^{b}, \\
& B^{c i}{ }_{\mid i}^{c}=-\Lambda_{a b}^{c} \gamma^{a b}=-\gamma^{c g} C^{a}{ }_{g a}=-2 a^{c} .
\end{aligned}
$$

\footnotetext{
${ }^{7}$ A more common choice is $\left[\boldsymbol{B}_{a}, \boldsymbol{n}\right]=0$.
} 
For reference we list the forms of the quantities used earlier,

$$
\begin{aligned}
g_{i j}= & \gamma_{a b}(t) B_{i}^{a} B_{j}^{b} \\
V_{i}= & V_{a} B_{i}^{a}\left(V_{a} \text { constants }\right) \\
K_{i j}= & \frac{1}{2 N}\left(V^{g}\left(C_{a g b}+C_{b g a}\right) e^{2 \phi}+\dot{\gamma}_{a b}\right) B_{i}^{a} B_{j}^{b} \\
K= & \frac{1}{2 N}\left(4 V^{g} a_{g} e^{2 \phi}+\gamma^{a b} \dot{\gamma}_{a b}\right) \\
N= & e^{\phi}\left(1+\gamma^{a b} V_{a} V_{b} e^{2 \phi}\right)^{1 / 2} \\
\Omega= & K_{a b} K_{c d}\left(\gamma^{a c} \gamma^{b d}-\gamma^{a b} \gamma^{c d}\right) \\
\dot{u}_{j}= & \phi_{, 4} V_{a} B_{j}^{a} ; \dot{u}_{4}=0 \\
\omega_{i j}= & -e^{\phi} C^{c}{ }_{a b} V_{c} B_{i}^{a} B_{j}^{b} ; \omega_{4 v}=0 \\
\omega^{i}= & \frac{e^{2 \phi}}{N}\left(n^{a d}+\eta^{d b a} a_{b}\right) V_{a} B_{d}^{i} ; \omega^{4}=V_{a} \omega^{a} \\
\sigma_{i j}= & \frac{N^{1 / 3} e^{-4 \phi / 3}}{2}\left(\frac{\gamma_{a b}+e^{2 \phi} V_{a} V_{b}}{\left(N e^{-\phi}\right)^{1 / 3}}\right)_{, 4} B_{i}^{a} B_{j}^{b} \\
R_{i k}^{*}= & \left(2 n_{a}{ }^{g} n_{g b}-n_{d}^{d} n_{a b}-2 \eta_{c d(a} n_{b}{ }^{c} a^{d}\right. \\
& \left.-\gamma_{a b}\left(2 a^{d} a_{d}+n^{f s} n_{f s}-\frac{\left(n^{d}{ }_{d}\right)^{2}}{2}\right)\right) B_{i}^{a} B_{k}^{b} \\
R^{*}= & -6 a^{d} a_{d}-n^{g t} n_{g t}+\frac{1}{2}\left(n_{d}^{d}\right)^{2}
\end{aligned}
$$

where $\dot{\gamma}_{a b}=d \gamma_{a b} / d t$.

The field Eqs. (2.5), (2.6), (2.8), (2.9) become

$$
\begin{aligned}
& \frac{1}{2}\left(-R^{*}+\Omega\right)=p-(w+p) N^{2} e^{-2 \phi} \\
& K_{b}^{a} C_{a d}^{b}-K_{d}^{a} C_{g a}^{g}=(w+p) N V_{d}=\frac{s_{0} V_{d}}{\sqrt{\gamma}} \\
& \frac{K_{, 4}}{N}+K_{a b} K^{a b}=\frac{w-p}{2}-(w+p) N^{2} e^{-2 \phi} \\
& R_{b}^{* a}+\frac{\left(\sqrt{\gamma} K_{b}^{a}\right)_{, 4}}{N \sqrt{\gamma}}+\frac{2\left(p^{d} a_{d}\right) K_{b}^{a}}{N}-\left(K^{a}{ }_{f} C_{b d}^{f} p^{d}+K_{b}^{f} C_{d f}^{a} p^{d}\right) \\
& =(w+p) e^{2 \phi} V^{a} V_{b}+\left(\frac{w-p}{2}\right) \delta_{b}^{a}
\end{aligned}
$$

where $s_{0}=s N \sqrt{\gamma} e^{-\phi}=N(w+p) \sqrt{\gamma}$ is a constant.

From (2.11) we find that

$$
\left(\sqrt{\gamma}\left(K_{b}^{a} C^{b}{ }_{a d}-K_{d}^{a} C_{g a}^{g}\right)\right)^{\cdot}=0
$$


as (3.12) also shows. If (3.12) holds then (2.10) implies

$$
\left(M n^{\varrho}\right)_{; \varrho}=0
$$

where $M=N^{2} M^{44}=\left(\frac{1}{2}\left(\Omega-R^{*}\right)-p+(w+p) N^{2} e^{-2 \phi}\right) N^{2}$ and since the initial value is $M=0$ we have $M=0$ at all times, so that (3.11) is conserved, i.e., is a first integral.

\section{Lagrangian Principles in Spatially-Homogeneous Universes}

If we apply the process leading from (1.7) to (1.9), the action (2.13) gives

$$
I=\int\left(R^{*}+\Omega+2 p\right) N \sqrt{\gamma} d t ;
$$

the $L\left(x^{i}\right)$ that appears in (1.8) being $\operatorname{det} B_{i}^{a}$. One can derive ten equations from the time integrand by varying $\gamma_{a b}, p^{a}$ and $N$. Varying $p^{a}$ is equivalent to varying the velocity of the fluid relative to observers moving along the hypersurface-normal congruence, while varying $N$ is equivalent to varying the energy density of the fluid. (Note that if one uses the normal congruence, $p^{f}=0$ and $N=e^{\phi}$ then one can vary only the $\gamma_{a b}$, getting only six equations [11].) Misner has followed essentially this same procedure, giving Lagrangian and Hamiltonian forms for the field equations of universes of Bianchi types I and IX, but using $I_{3}$ of (2.14) $[12-14]$. This procedure was generalized by Hawking [11] to all models of Class A of [1], i.e., all those in which $a^{b}=0$. Further generalizations and applications are in Refs. [15-19], etc.

However, the ten equations we get are not always the correct Einstein field equations for perfect fluid, as was first realized by Hawking [11]. The reason is that the unwanted terms arising from (2.15)-(2.18) are in general non-vanishing. From (3.9) any spatial divergence of an invariantly defined quantity has the form

$$
\left(\sqrt{g} F^{i}\right)_{, i}=\left(F^{a} B_{a}{ }^{i}\right)_{i i} \sqrt{g}=-2 F^{g} a_{g} \sqrt{g} .
$$

Explicitly when variations are taken the terms (2.15)-(2.18) give rise to the unwanted extra time integrals

$$
\begin{gathered}
\int N \sqrt{\gamma} C^{f}{ }_{g f}\left(C^{b}{ }_{n b} \gamma^{n a} \gamma^{g b}-C^{b}{ }_{n r} \gamma^{a r} \gamma^{g n}\right) \gamma_{a b}^{\prime} d t \\
=\int N \sqrt{\gamma}\left(6 a^{g} a^{h}-2 a^{f} \eta_{f}{ }^{g}{ }_{t} n^{h) t}-2\left(a_{f} a^{f}\right) \gamma^{g h}\right) \gamma_{g h}{ }_{g h} d t \\
0 \\
\int 4 \sqrt{\gamma} a_{g}\left(K^{g}{ }_{f}-K \delta^{g}{ }_{f}\right)\left(p^{f}\right)^{\prime} d t, \\
\int 2 \sqrt{\gamma} p^{d} a_{d}\left(K^{g f}-K \gamma^{g f}\right) \gamma_{g f}^{\prime} d t .
\end{gathered}
$$


The first result is that the Lagrangian given by (4.1) gives the correct equations if and only if

$$
a^{g}=0
$$

that is, if and only if the space is of Class A of $[1]^{8}$.

Clearly, the terms all vanish if $a^{g}=0$, by (4.2). Suppose the terms do vanish. The coefficient of $\left(p^{f}\right)^{\prime}$ must vanish, so that

$$
a_{g}\left(K_{f}^{g}-K \delta_{f}^{g}\right)=0 .
$$

If we now take the coefficient of $\gamma_{a b}^{\prime}$ and contract it on $a_{b} a_{a}$, this, which would vanish if the coefficient vanished, is $4\left(a_{d} a^{d}\right)^{2}$. Thus we see that (4.6) must hold.

Next we prove that the same is true for any Lagrangian differing from (4.1) by addition or subtraction of time integrals - in particular for the specialization of $I_{1}$ or $I_{3}$ in the same manner already described for $I_{2}$. That is to say, the sum of the terms (4.2)-(4.5) are not the variation of some integral.

We prove this by considering the integrand of (4.1) formally as a differential form $\omega$ in the space of $\left(N, p^{f}, \gamma_{a b}\right)$ so we replace $p^{f^{\prime}}$ by $d p^{f}$ etc. The integrability conditions can then be determined by calculating $d \omega$. From the $d N \wedge d p^{c}$ terms we find the conditions (4.7). The $d N \wedge d \gamma_{a b}$ term then gives (4.6) by contraction with $a^{a} a^{b}$.

It should be noted that these conclusions are in fact independent of the matter content of the space. All the unwanted terms we have been discussing arise from the geometrical part of the Lagrangian and for these terms the congruence of fluid worldlines has no particular physical significance. That is, we could always choose to refer the equations to a timelike congruence obeying the perfect fluid equations of motion and (3.3), regardless of whether the actual matter content was a perfect fluid flowing along that congruence.

One can now embark on an extensive investigation of when one can obtain some, though not all, of the Einstein equations for Class B spaces correctly from a variational principle, the remaining equations being imposed as extra conditions. Few of the many possibilities make practical sense, but one, which we consider next, does. This is to try to obtain the "evolution equations" (3.14) (or rather the corresponding equations in $G_{a b}$ ), since it is known that (3.11) and (3.12), the "Hamiltonian constraint" and "dynamical constraints" are first integrals of (3.14). We may attempt this for the case $p^{d}=0$, since it appears the natural simple choice. We can show, by a method similar to the above proofs for the general case, that this will not in general yield correct equations for

${ }^{8}$ One could say that the natural boundary conditions for (4.1) are that the space-time is of Class A. 
Class B spaces. When the variations are taken we get

$$
I^{\prime}=\int\left(E^{a b}+U^{a b}\right) \gamma_{a b}^{\prime} d t
$$

where the Einstein equations are $E^{a b}=0$ and the unwanted term $U^{a b} \gamma_{a b}^{\prime}$ is given by (4.3). Again $U^{a b} a_{a} a_{b}=4 N \sqrt{\gamma}\left(a_{d} a^{d}\right)^{2}$ so $U^{a b} \gamma_{a b}^{\prime}$ is non-vanishing in general. Moreover if $d\left(U^{a b} d \gamma_{a b}\right)$ is taken (in the $\gamma_{a b}$ space) we find we get $U^{a b c d}\left(d \gamma_{a b} \wedge d \gamma_{c d}\right)$ and $U^{a b c d} a_{a} a_{b} \gamma_{c d}=\sqrt{\gamma}\left(a_{d} a^{d}\right)^{2}$, which is nonvanishing in Class $B$ spaces. Thus neither (4.1) nor any Lagrangian differing from it by addition or subtraction of terms gives the evolution equations correctly.

We may investigate a little further. $U^{a b}$ is a traceless symmetric tensor and so has only two independent eigenvalues. Thus, at each time the variation (4.8) gives four correct Einstein equations. We can sort these out by introducing a basis of orthonormal eigenvectors of $n_{a b},\{\boldsymbol{e}\}$, of which $\stackrel{1}{e}$ is parallel to $a_{b}$. (These are just the basis used in [1].) We find that the eigenvectors of $U^{a b}$ are $\stackrel{1}{\boldsymbol{e}}, \boldsymbol{X}=\frac{1}{2}(\stackrel{2}{\boldsymbol{e}}+\stackrel{3}{\boldsymbol{e}})$ and $\boldsymbol{Y}=\frac{1}{2}(\stackrel{2}{\boldsymbol{e}}-\stackrel{3}{\boldsymbol{e}})$ and these have differing eigenvalues in general. Thus the variations $\gamma_{a b}^{\prime}=\alpha(t) \gamma_{a b}$ $+\beta(t) \stackrel{1}{e}_{(a} X_{b)}+\gamma(t) \stackrel{1}{e}_{(a} Y_{b)}+\delta(t) X_{(a} Y_{b)}$ give correct field equations and we need to find the two equations $E^{a b} e_{a}^{1} e_{b}^{1}=0$ and $E^{a b} X_{a} X_{b}=0$ (or equivalently $E^{a b} Y_{a} Y_{b}=0$ ) by some other means.

One linear combination of these equations may be obtained by requiring that all three of Eqs. (3.12) hold. That is by imposing Eqs. (3.12) as a constraint. It is a consequence of the fact that Eqs. (3.12) are equivalent to

$$
\left(C_{a d}^{b}-\delta_{d}^{b} C_{g a}^{g}\right) E_{b}^{a}=0
$$

and the fact that

$$
\left(C_{a d}^{b}-\delta_{d}^{b} C_{g a}^{g}\right) U_{b}^{a}=2 a_{d}\left(12 a_{f} a^{f}+2 n^{f s} n_{f s}-\left(n_{s}^{s}\right)^{2}\right)
$$

that this requirement gives us only one additional correct combination of the Einstein field equations in a Class B space in addition to the four obtained by restricted variations of the type given above.

The problem is to find any neat way of regaining the sixth evolution equation. One can of course derive it from Eq. (3.11) by differentiation, but apart from this there appears to be no more elegant and practical way, in the general case, than simply imposing it as a constraint. In two special cases we can get over this problem. The first is when the two eigenvalues of $n^{a b}, n_{2}$ and $n_{3}$ [1], are equal, for then $U^{a b}$ has only one independent eigenvalue. However we already know that in the case $p^{d}=0, n_{2}=n_{3}$ implies the space is Robertson-Walker. The second case is when $n^{a}{ }_{a}=0$. The derivatives of this constraint give us (among other 
things) the missing Einstein equation. If we substitute $n^{a}{ }_{a}=0$ and its consequences into the Lagrangian and vary only within the restricted set remaining, we get the field equations for the remaining undetermined quantities (at least, when $p^{f}=0$ ) [16] and this can be extended to fluids with anisotropic stress [15].

Rather than pursue similar investigations for other special variational principles we turn now to the cases where the general form holds good, the Class A models.

\section{The Variational Principle for Space-Times with Class $A$ Groups}

In this section we shall evaluate the Lagrangian function when the isometry group is of Class $\mathrm{A}$, that is when

$$
a_{a}=0 \text {. }
$$

In this case the constants of structure of the group may be written as

$$
C_{b c}^{a}=\eta_{b c t} n^{a t}=\varepsilon_{b c t} m^{a t}
$$

where the $m^{a t}$ are the coefficients of a constant symmetric matrix and

$$
m^{a t}=m^{t a}=\sqrt{\gamma} n^{a t} .
$$

Hence Eq. (3.10) may be written as

or

$$
\begin{aligned}
& \sqrt{\gamma} R^{*}=\sqrt{\gamma}\left(\frac{\left(n_{a}^{a}\right)^{2}}{2}-n^{c d} n_{c d}\right) \\
& \sqrt{\gamma} R^{*}=\frac{\gamma_{a c} \gamma_{b d}}{2 \sqrt{\gamma}}\left(m^{a c} m^{b d}-2 m^{a b} m^{c d}\right) .
\end{aligned}
$$

The Eqs. (3.10) defining $K_{a b}$ become

$$
K_{a b}=\frac{1}{2 N}\left(\dot{\gamma}_{a b}+p^{g}\left(\eta_{g b t} n_{a}^{t}+\eta_{g a t} n_{b}^{t}\right)\right) .
$$

Hence

$$
K=\gamma^{a b} K_{a b}=\frac{1}{2 N} \gamma^{a b} \dot{\gamma}_{a b}=\frac{1}{N} \frac{(\sqrt{\gamma})}{\sqrt{\gamma}}
$$

and

$$
\begin{aligned}
K_{a b} K^{a b}= & \frac{1}{4 N^{2}}\left\{\dot{\gamma}_{a b} \dot{\gamma}_{c d} \gamma^{a c} \gamma^{b d}+4 p^{g} \dot{\gamma}_{a b} \gamma^{b c} \varepsilon_{g c t} m^{a t}\right. \\
& \left.-4 p^{g} p_{g} R^{*}+\frac{4 \gamma_{a b} p_{c} p_{t}}{\gamma}\left(m^{a b} m^{c t}-\frac{3}{2} m^{c b} m^{t a}\right)\right\} .
\end{aligned}
$$

Since the spatial components of the vorticity vector are given by

$$
\omega^{d}=\frac{e^{2 \phi}}{N} V_{a} n^{a d}=\frac{p_{a} n^{a d}}{N}=\frac{p_{a} m^{a d}}{N \sqrt{\gamma}}
$$


we may write

$$
\begin{aligned}
\Omega=\Omega_{0} & +\frac{p^{g}}{N^{2}} \dot{\gamma}_{a b} \gamma^{b c} \varepsilon_{g c t} m^{a t}-\frac{p^{g} p_{g}}{N^{2}} R^{*} \\
& +\frac{\gamma_{a b} m^{a b}}{N \sqrt{\gamma}} p_{c} \omega^{c}-\frac{3}{2} \gamma_{a b} \omega^{a} \omega^{b}
\end{aligned}
$$

where

$$
\Omega_{0}=\frac{1}{4 N^{2}}\left(\gamma^{a b} \gamma^{c d}-\gamma^{a c} \gamma^{b d}\right) \dot{\gamma}_{a c} \dot{\gamma}_{b d} .
$$

Thus

$$
I=\int\left(R^{*}+\Omega+2 p\right) N \sqrt{\gamma} d t
$$

may be written as

$$
I=\int\left(\Omega_{0}-V+2 p+\mathscr{L}_{i}\right) \sqrt{\gamma} d \tau
$$

where $\tau$ is defined by the equation

$$
d \tau=N d t
$$

and measures the cosmic time, we have written

$$
\begin{aligned}
\Omega_{0} & =\frac{1}{4}\left(\gamma^{a b} \gamma^{c d}-\gamma^{a c} \gamma^{b d}\right) \gamma_{a c}^{+} \gamma_{b d}^{+} \\
-V & =\frac{e^{2 \phi}}{N^{2}} R^{*}+\frac{\gamma_{a b} m^{a b}}{N \sqrt{\gamma}} p_{c} \omega^{c}-\frac{3}{2} \gamma_{a b} \omega^{a} \omega^{b} \\
\mathscr{L}_{i} & =\frac{p^{g}}{N} \gamma_{a b}^{+} \gamma^{b c} \varepsilon_{g c t} m^{a t}=\frac{p^{g}}{N} \gamma_{a b}^{+} \gamma^{c b} C_{g c}^{a}
\end{aligned}
$$

and we have used the notation

$$
\gamma_{a b}^{+}=\frac{1}{N} \dot{\gamma}_{a b}=\frac{d \gamma_{a b}}{d \tau} .
$$

When $p_{a}=e^{2 \phi} V_{a}=0$, Eq. (5.6) reduces to

$$
I=\int\left(R^{*}+\Omega_{0}+2 p\right) \sqrt{\gamma} d \tau
$$

and determines a variational principle from which one may derive the Einstein equations in case the world lines of the fluid are orthogonal to the invariant varieties. When this is not the case the Lagrangian is modified by the introduction of the term $\mathscr{L}_{i}$ and by the replacement of $R^{*}$ by $-V$ (cf. [18]). Thus when the world lines of the fluid are not orthogonal to the invariant varieties, the dependence of the Lagrangian on $\gamma_{a b}$ changes by the modification of $R^{*}$ by the factor $\left(1+p^{a} p^{b} \gamma_{a b}\right)^{-2}$, and the addition of terms linear and quadratic in the vorticity vector $\omega^{a}$. In addition the term $\mathscr{L}_{i}$ which is linear in $\gamma_{a b}^{+}$, appears.

The Einstein field equations are obtained from Eq. (5.6) by varying $\gamma_{a b}, N$ and $P^{a}$ independently. Under such a variation we have

$$
\phi^{\prime}=e^{-2 \phi}\left(N^{\prime} N-p_{a} p^{\prime a}-\frac{1}{2} \gamma_{a b}^{\prime} p^{a} p^{b}\right) .
$$


The six equations obtained by varying the $\gamma_{a b}$ keeping $N$ and $p^{a}$ fixed have as first integrals the four equations obtained by varying $N$ and $p^{a}$ keeping $\gamma_{a b}$ fixed. Hence we may regard Eq. (5.6) as determining a variational principle in which the $\gamma_{a b}$ alone are varied and $N$ and $p^{a}$ are kept fixed.

The equations obtained by varying $p^{a}$ are Eqs. (3.12) and may be written as

$$
\begin{aligned}
\frac{1}{N} \gamma_{a b}^{\cdot} \gamma^{b c} \varepsilon_{g c t} m^{a t}=2 \frac{p_{g} R^{*}}{N^{2}} & -2 \frac{\gamma_{a b} m^{a b}}{N \sqrt{\gamma}} \omega_{g}+3 \frac{\omega_{a} m^{t a}}{N \sqrt{\gamma}} \gamma_{t g} \\
& -2 \frac{s_{0} V_{g}}{N \sqrt{\gamma}} .
\end{aligned}
$$

Hence Eq. (5.10) may be written as

$$
\begin{aligned}
\mathscr{L}_{i}=2 \frac{p_{g} p^{g}}{N^{2}} R^{*} & -2 \frac{\gamma_{a b} m^{a b}}{N \sqrt{\gamma}} \omega_{g} p^{g}+3 \omega_{a} \omega^{a} \\
& -2 \frac{s_{0} e^{-2 \phi} p_{g} p^{g}}{N \sqrt{\gamma}}
\end{aligned}
$$

and may be used to rewrite Eq. (5.6).

The variation of the integral given by Eq. (5.6) may be performed by replacing the varied functions $N, p^{a}$ and $\gamma_{a b}$ by another equivalent set such as $\phi, p_{a}$ and $\gamma_{a b}$. The result of this replacement is to replace Eqs. (3.11) to (3.14) by an equivalent set of equations. We may choose the quantities to be varied so as to take advantage of any information available in a given problem; for example we may fix the initial conditions of the problem and hence keep the $V_{a}$ as fixed constants and not vary them while determining the $\phi$ and $\gamma_{a b}$ which extremalize the integral given by Eq. (5.6). Eqs. (3.12) (that is, (5.12)) and (3.11) may then be regarded as equations determining some of the unknown functions, $\gamma_{a b}$ and $\phi$ (cf. [17]).

\section{Conclusions}

We have shown above that the general action principles for Einstein's equations are not valid for Class B spatially-homogeneous models if the spatial-homogeneity is imposed before the variations are made. The reason is that the allowed variations in the general principle are variations which vanish at the boundary of a compact region, while spatiallyhomogeneous variations which vanish at such a boundary would vanish everywhere, and so are not of the allowed kind. This means that partial integral terms which normally vanish due to the vanishing of the variation at the boundary, may be non-zero in the spatially homogeneous case. 
The same difficulty could recur in other situations where it was desired to impose symmetry of the solution on a Lagrangian action before taking the variations of that action. In particular one would need to check that any proposed Lagrangian for plane or cylindrically symmetric space-times did not suffer from this defect.

The invalidity of Lagrangian principles is equally an invalidity of Hamiltonian principles, since one can always construct the one from the other. Nor does the compactification of the space sections, which might be thought to help because one need no longer have a spatial boundary, make any difference. The reason for this last is that the principle appealed to is supposed to be valid for any compact region, and this is false. The invalidity becomes manifest, of course, when incorrect Einstein equations are derived, but if one is unware of the trap one may fail to check this.

The virtue of the action principle when it does exist is that one can easily change one's choice of variables by simply substituting the new variables in the action. This is often simpler than directly substituting into the Euler-Lagrange equations. Similarly, one can study restricted subsets of the solutions by substituting the constraint into the Lagrangian and calculating the relevant equations for the constrained subset. In addition it is often easier to understand qualitative features of the solutions by considering the action principle. As we have shown, these advantages are not necessarily available to us in spatially-homogeneous models.

The derivation of the matter terms in the field equations from a matter Lagrangian could, in principle, meet with difficulty in the spatiallyhomogeneous case even when the geometric terms do not. This has not, however, been found to happen in the various cases that have been investigated $[11,16-19]$. Indeed rather general positive results about the existence of such Lagrangians can be deduced from the spatial-homogeneity assumptions when $n_{\mu} T^{\mu v} k_{v \sigma}=0[15]$.

\section{References}

1. Ellis, G.F.R., MacCallum, M.A.H.: Commun. math. Phys. 12, 108 (1969).

2. Taub, A. H.: Proceedings of the 1967 Colloque on "Fluids et champ gravitationnel en relativité générale", No. 170, 57, Paris: Centre National de la Recherche Scientifique (1969).

3. Schutz, B. F., Jr.: Phys. Rev. D 2, 2162 (1970).

4. Treciokas, R., Ellis, G. F. R.: Commun. math. Phys. 23, 1 (1971).

5. Ehlers, J.: Akad. Wiss. Lit. (Mainz), Abh. Math.-Nat. Kl. No. 11 (1961).

6. Arnowitt, R., Deser, S., Misner, C. W.: Chapter 7 of "Gravitation: an introduction to current research" ed. L. Witten. New York: Wiley 1962. 
7. Hicks, N.J.: "Notes on Differential geometry" Van Nostrand. Mathematical Studies No. 3, Princeton: van Nostrand 1965.

8. Kantowski, R., Sachs, R. K.: J. Math. Phys. 7, 443 (1966).

9. Taub, A. H.: Ann. Math. 53, 472 (1951).

10. Estabrook, F., Wahlquist, H. D., Behr, C. G.: J. Math. Phys. 9, 497 (1968).

11. Hawking, S. W.: Monthly Notices Roy. Astron. Soc. 142, 129 (1969).

12. Misner, C. W.: Astrophys. J. 151, 431 (1968).

13. - Phys. Rev. Letters 22, 1071 (1969).

14. - Phys. Rev. 186, 1319 (1969).

15. MacCallum, M.A.H., Stewart, J.M., Schmidt, B. G.: Commun. math. Phys. 17, 343 (1970).

16. - Commun. math. Phys. 20, 57 (1971).

17. Ozsvath, I.: J. Math. Phys. 11, 2860 (1970).

18. Ryan, M.P.: Univ. of Maryland Tech. Rpt. $70 \sim 046$ (1970).

19. Matzner, R. A.: Commun. math. Phys. 20, 1 (1971).

\author{
A. H. Taub \\ Dept. of Mathematics \\ University of California \\ Berkeley, Calif. 94720, USA
}

